\title{
PLANAR SLOT-PATCH ANTENNA
}

\author{
Jan Zehentner, Jan Machac, Petr Lorenz, Jan Mrkvica \\ Czech Technical University, Technicka 2, 16627 Prague 6, Czech Republic \\ tel. +420 2 24352273, fax +420224355865, e-mail zeh@ cesnet. cz
}

\begin{abstract}
This paper discusses investigations of leaky wave radiation from the slotline made on a low permittivity substrate. New findings on the field distribution of the space leaky wave propagating on a slotline with a wide slot are presented. The design of the antenna and its measured parameters are introduced, along with practical experience acquired experimentally.
\end{abstract}

\section{INTRODUCTION}

Low profile and entirely planar antennas have attracted strong interest of researchers and designers for more than thirty years. Individual radiators are mostly grouped into one- or two-dimensional arrays to produce the desired radiation pattern, to scan space or to increase the gain of the antenna. The main advantage of such a planar antenna arrangement is that it saves space and enables the antenna to be located on the external surface of a body, according to its shape. Many kinds of planar antennas are already known and have been successfully used, particularly in communications ranging from the $\mathrm{D}$ to $\mathrm{L}$ band. To produce radiation in the narrow or wide frequency band, either the standing wave in the resonant structure or the traveling wave on the transmission line is utilized, Bahl and Bhartia (1), Tzuang (2). The latter case is treated in this paper.

We have investigated the behaviour of the slotline in the wide frequency band and determined its dispersion characteristics. It has turned out that the space leaky wave can excite and radiate on a slotline with a sufficiently wide slot. A new view is introduced on the propagation constants referring to the space leakage obtained from the dispersion equation. The aim of this paper is to evaluate the ability of the slotline to radiate, to examine the far field, to design and measure the antenna, and to suggest possible applications.

\section{SPACE LEAKY WAVES ON THE SLOTLINE}

In order to calculate the dispersion characteristics of the slotline, a cross-section of which is shown in Fig. 1, we used the method of moments in the spectral domain. The complex propagation constants of waves transmitted by the slotline on a substrate with permittivity 2.6, a thickness of $1.2 \mathrm{~mm}$ and a slot width of $60 \mathrm{~mm}$ are plotted in Fig. 2. They belong to the dominant bound wave with even symmetry of the transversal component of the electric field within the slot, to the 1st higher order bound wave, the 1st and 3rd space leaky wave with odd symmetry, and to the 2nd space leaky wave with even symmetry. Since the 2 nd higher order bound wave with even symmetry and also all surface leaky waves may occur at frequencies higher than $15 \mathrm{GHz}$ they are not included in Fig. 2.

Zehentner and Machac (3) gave an overview and classification of the dominant and higher order bound waves, and of the space leaky waves of the 1 st and 2 nd class with odd and even electric field symmetry within the slot. Further recent research into solutions of the dispersion equation of the slotline has resulted in a reclassification of space leaky waves as follows. Unlike (3) there is now only one category of space leaky waves. Passing from one characteristic to another, the minimum of the normalized phase constant and the entire corresponding normalized leakage constant displace towards the higher frequencies. Fig. 3, in which a number of solutions of the dispersion equation are plotted, makes the situation clearer. The leftmost line was computed with one and one, or three and three basis functions in the $\mathrm{x}$ and $\mathrm{z}$ direction, respectively. The remaining lines originated when only one and one basis function was accounted for. The shift of particular characteristics is more distinct when a higher permittivity substrate is used. Space leaky waves corresponding to particular branches of dispersion characteristics are of the first order according to their field distribution within the slot. With the growing branch number the field in the space is more and more complex with very rapidly growing amplitude. This is documented by Fig. 4, where the electric field distribution of waves corresponding to the first and second branches of the dispersion characteristics in Fig. 3 are given. We confirm the brief notification regarding the number of solutions made in (2) with respect to the suspended microstrip, i.e., there should be infinitely many of these solutions. Any solution is physically meaningful when the corresponding leakage constant is sufficiently low. Mostly published works refer to the leftmost solution denoted in Fig. 2 as the first space leaky wave propagation constant. A high leakage constant does not result in high radiation efficiency. It indicates enhanced leakage of power into the substrate. For these practical reasons, only the above-mentioned solution with the highest phase constant and lowest leakage constant at low frequencies is utilized for operating a slotline leaky wave antenna. 


\section{SLOTLINE LEAKY WAVE ANTENNA}

The antenna design was based on the background presented above. A microstrip patch placed along the center line of the slotline on the opposite side of the substrate excites the first space leaky wave, as in Sheen and Lin (4). The feeder is unable to excite the 2nd and 3rd space leaky wave, since their fields and the feeder field are not in conformity with each other. However, the field of the 1st higher order bound wave resembles the field of the 1st space leaky wave and they can excite simultaneously. Mapping the field by a small probe movable across and along the slot confirmed domination of the 1st space leaky wave in the excited spectrum. The feeder dimensions were designed by means of the Zeland IE3D simulator. The length of the slotline was determined by the distance at which the amplitude of the 1st space leaky wave decreased to zero.

The orientation of the co-ordinates and the slotline used in radiation pattern measurement is sketched in Fig. 5. The measured and simulated radiation pattern $\mathrm{E}_{\theta}(\theta)$ of the slotline antenna in the vertical plane $\phi=0$ at $3.25 \mathrm{GHz}$ are shown in Fig. 6. Ripples in the radiation pattern arose due to undesired standing waves occurring in the metallized area. They disappear at lower frequencies, e.g., $2.5 \mathrm{GHz}$. Radiation pattern $\mathrm{E}_{\theta}(\phi)$ in the plane of the highest radiation inclined by 28 degrees from the horizontal plane is plotted in Fig. 7. The main beam inclined from 36.3 to 22.7 degrees when the frequency changed from 2.5 to $4.0 \mathrm{GHz}$. A $\chi$ onductor-backed slotline antenna exhibited half-space radiation with wider main beam and $-10 \mathrm{~dB}$ side lobes.

The second designed feeder was a short section of the CPW, the central conductor patch of which excited the first space leaky wave. Fig. 8 shows good agreement of the calculated and measured return losses. The co-ordinates and the antenna are sketched in Fig. 9. The original narrow slot terminating the antenna in the former case is now replaced by a short-circuit resulting in lower backward radiation. Analysis of the measured electric field along the edge of the slot confirmed the presence of the first higher order bound wave. Its reflection from the end of the slotline caused ripples in the radiation pattern. The matched load at the end of the antenna canceled the standing waves along the slot and removed ripples in the far field. The measured radiation pattern in Fig. 10 confirms this finding. The measured antenna efficiency was $7 \mathrm{~dB}$. The main beam inclined from 49 to 59 degrees when the frequency changed from 2.35 to $3.5 \mathrm{GHz}$.

\section{CONCLUSIONS}

The slotline with a wide slot on a thin and low permittivity substrate was investigated. Emphasis was put on radiation of power by the space leaky wave. The 1st, 2nd and 3rd space leaky waves were identified on the slotline. In addition, five consecutive solutions of the slotline dispersion equation belonging to the same category of constituent modes were revealed. The higher modes without doubt keep the field distribution within the slot, but have a more complex and a more rapidly growing field in space.

Excitation of the desired wave depends considerably on the feeder. Our antenna was designed for operation in the 2-4 Ghz band in order to avoid difficulties and expense connected with operation in the mm-wave band, where the advantages of this antenna type can be entirely utilized. The slotline antenna was fed by the microstrip patch placed on the metal-less side of the substrate. The measured return losses were lower than $-18 \mathrm{~dB}$ at operation frequency. Recent simulation of the feeder utilizing the CPW was very promising. It offers better antenna parameters and fully planar arrangement of the circuit and radiator. Field mapping across and along the slot confirmed the presence and domination of the first space leaky wave. The origin of disturbing ripples in the radiation pattern, the first higher order bound wave, was identified and practically removed by an absorber terminating the antenna. The slotline antenna radiates two main beams, on opposite sides of the substrate. Short circuited termination of the slot reduces backward lobes. Frequency scanning sensitivity $7.7 \mathrm{deg} / \mathrm{GHz}$ was achieved in the $2.5-4.0 \mathrm{GHz}$ range and $14.8 \mathrm{deg} / \mathrm{GHz}$ in the $2.35-3.5 \mathrm{GHz}$ range, respectively. Measured and calculated radiation patterns compare well. A conductor-backed slotline antenna radiates only to the half-space and could become a counterpart to the microstrip leaky wave antenna. The first space leaky wave, especially the first mode, is suitable for antenna operation.. Phased arrays can lead to beam forming.

\section{ACKNOWLEDGEMENT}

This work has been supported by the Grant Agency of the Czech Republic under project 102/00/0025 „Planar circuits for $\mathrm{mw}$ and mmw technology“ and by research programmes J04/98 212300014 „Information and Communication Technology“ and MSM 216200031 „Intensive Numerical Calculations and Parallel Programming“.

\section{REFERENCES}

[1] I. J. Bahl, P. Bhartia: Microstrip Antennas. Artech House, 1980 Dedham.

[2] C.-K. C. Tzuang: Leaky Mode Perspective of Printed Antenna. 1998 Asia-Pacific Microwave Conference, vol. 3, pp. 1471-1478, December 1998, Yokohama.

[3] J. Zehentner, J. Machac: New Space Leaky Wave Spectrum Components on the Slotline. Conference Proceedings of the 30th EuMC, vol. 2, pp. 190-193, June 2000, Paris.

[4] J.-W. Sheen, Y.-D. Lin: Propagation Characteristics of the Slotline First Higher Order Mode. IEEE Trans. Microwave Theory and Tech., vol. MTT-46. no. 11, pp. 1774-1781, Nov. 1998. 


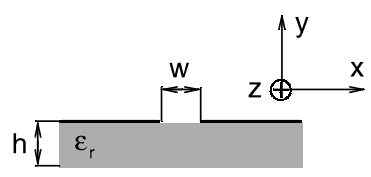

Fig. 1 Cross-section of the slotline

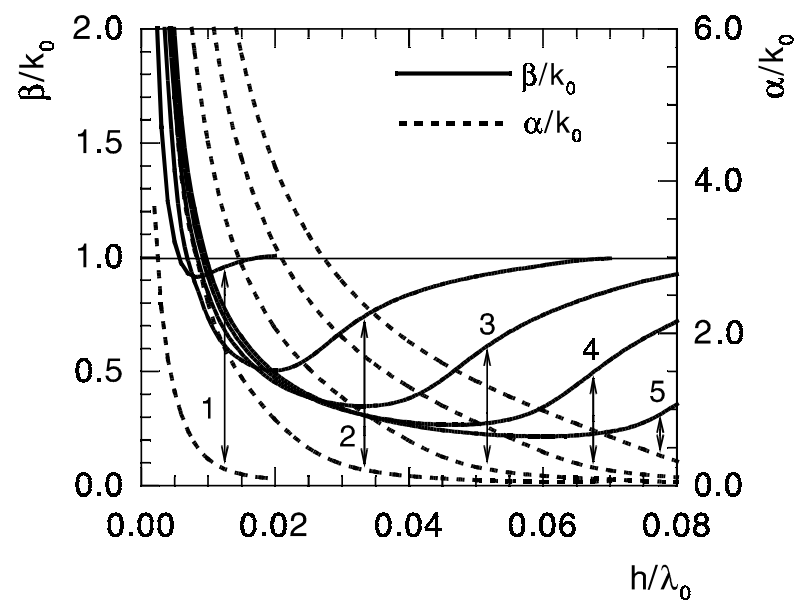

Fig. 3 Normalized phase $\beta / \mathrm{k}_{0}$ and leakage $\alpha / \mathrm{k}_{0}$ constant of the first space leaky wave on the slotline from Fig. 2 as the multivalue solution of the dispersion equation.

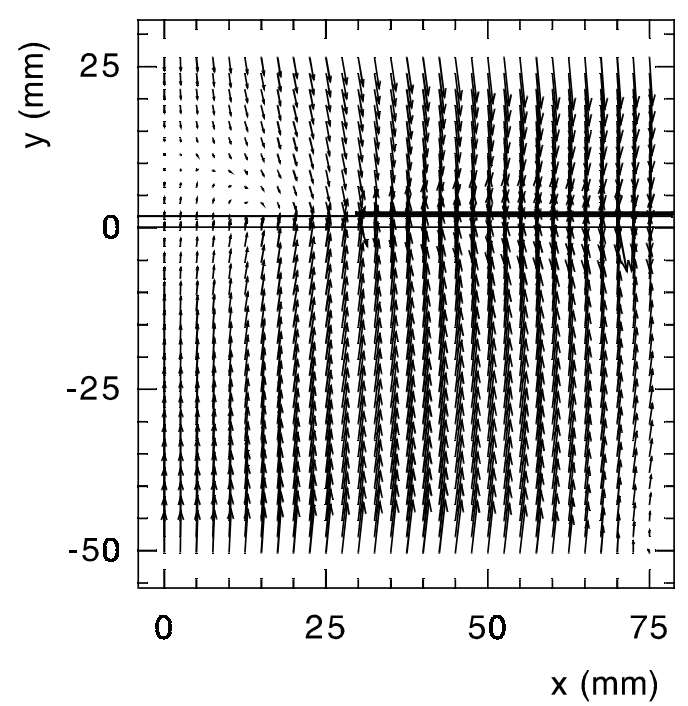

$\mathbf{a}$

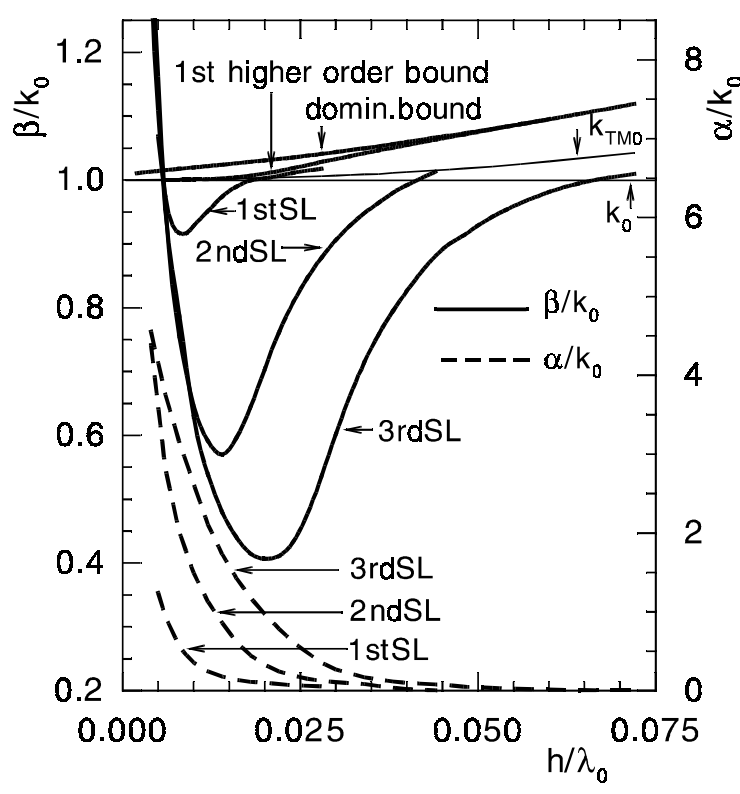

Fig. 2 Normalized phase $\beta / \mathrm{k}_{0}$ and leakage $\alpha / \mathrm{k}_{0}$ constant of the slotline with $\mathrm{w}=60 \mathrm{~mm}, \mathrm{~h}=1.2 \mathrm{~mm}$, $\varepsilon_{\mathrm{r}}=2.6$ depending on the normalized frequency $\mathrm{h} / \lambda_{0}$.

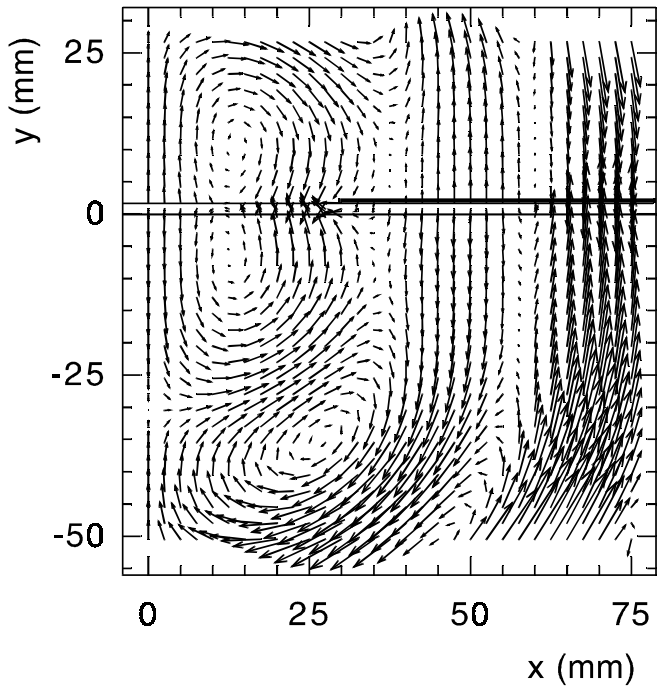

b

Fig. 4 Electric field distribution of the first space leaky wave corresponding to a) the first and b) the second branch of the dispersion characteristics from Fig. 3 at $h / \lambda_{0}=0.012$. 


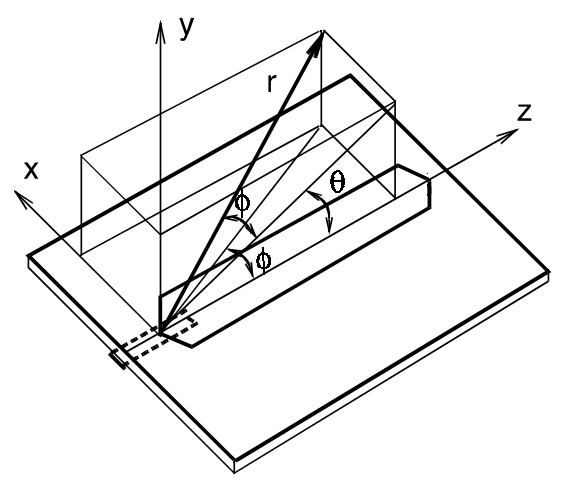

Fig. 5 Co-ordinates and the slotline antenna arrangement.

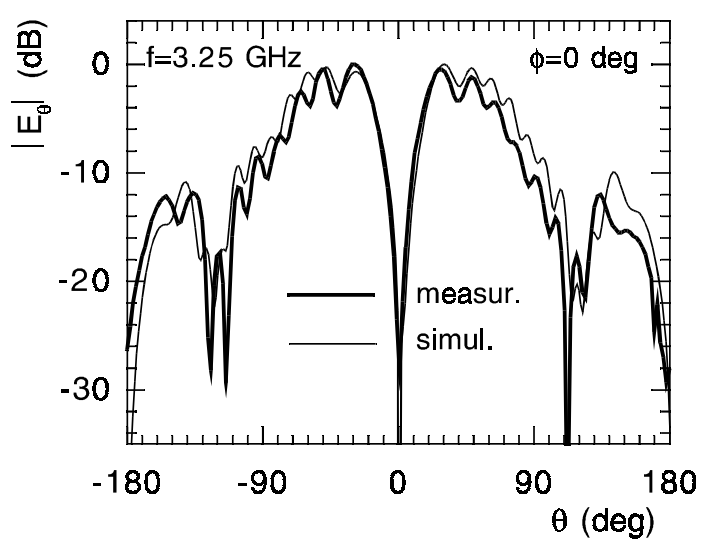

Fig. 6 Measured and simulated $\mathrm{E}_{\theta}(\theta)$ radiation pattern of the leaky wave slotline antenna in the plane $\phi=0$ degree at $3.25 \mathrm{Ghz}$.

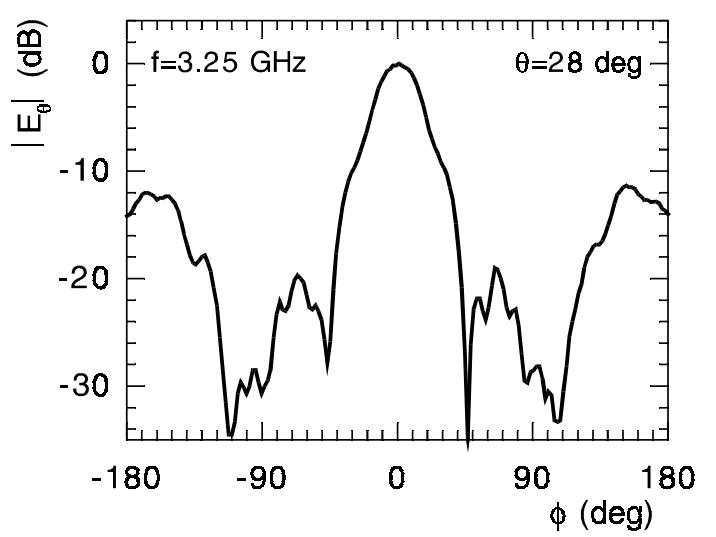

Fig. 7 Measured radiation pattern $\mathrm{E}_{\theta}(\phi)$ in the plane $\theta=28$ degree at $3.25 \mathrm{Ghz}$.

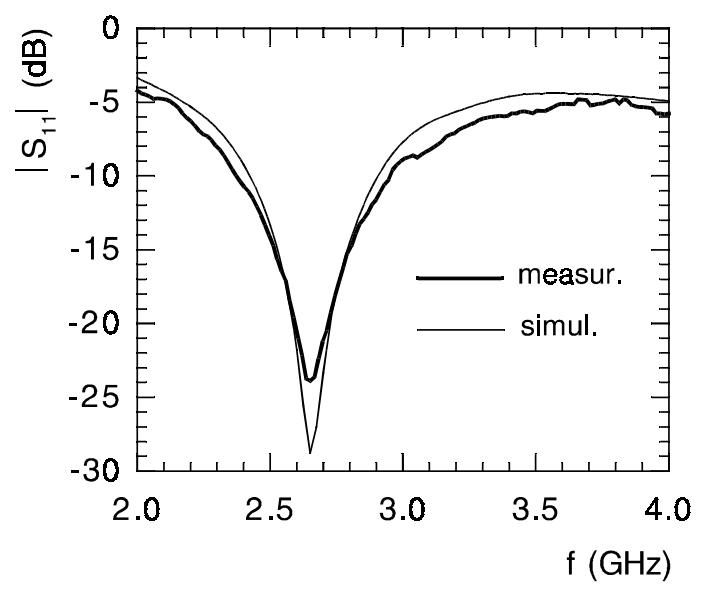

Fig. 8 Measured and simulated return loss of the slotline leaky wave antenna fed by the CPW.

Substrate thickness $1.2 \mathrm{~mm}$, permittivity 2.6 and slotwidth $60 \mathrm{~mm}$.

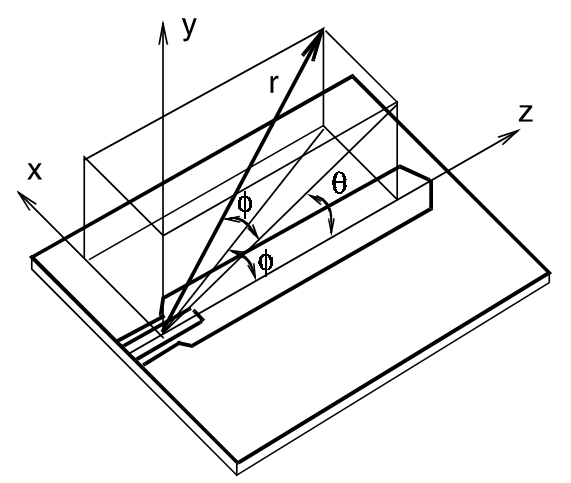

Fig. 9 Arrangement of the co-ordinates and the slotline antenna fed by the CPW.

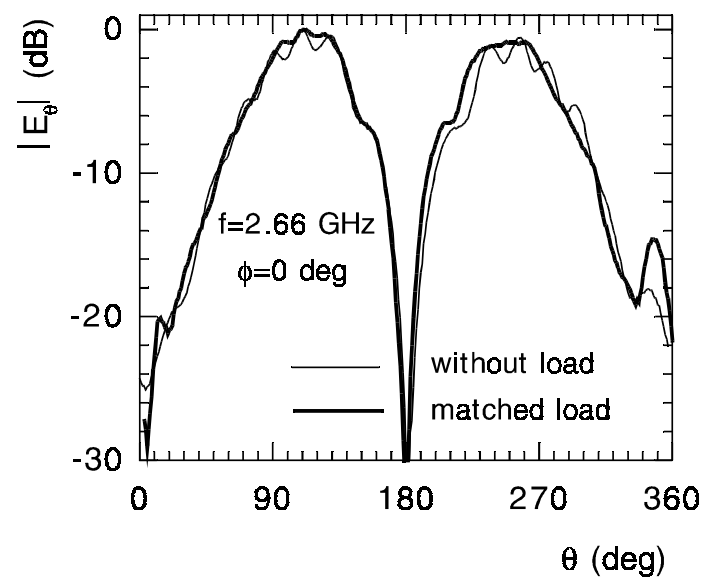

Fig. 10 Measured $E_{\theta}(\theta)$ radiation pattern of the leaky wave slotline antenna in the plane $\phi=0$ degree at 2.66

$\mathrm{GHz}$ when the CPW feeder was applied. The

rippleless pattern corresponds to the antenna terminated by an absorber. 